\title{
Evolution of Daily Activity Patterns from 1971 to 1981: A Study of the Halifax Activity Panel Survey
}

\author{
Andrew S. Harvey \\ Saint Mary's University \\ Halifax, Nova Scotia, Canada \\ Clarke Wilson \\ Canada Mortgage and Housing Corporation \\ Ottawa, Ontario, Canada
}

\begin{abstract}
Episode sequences from diaries are the richest source of information about daily activities of individuals and households available to social scientists. Their use has been advocated as an approach to urban planning that incorporates explicit consideration of the demands made by daily life on the built environment. The paper examines sequences of daily activities and activities augmented by data on their settings (including location and the presence of other people) to measure change in daily behaviour from 1971 to 1981. Diaries were supplied by respondents to the Halifax panel study carried out at Dalhousie University.

Episode sequences are analysed using alignment methods, also called optimal matching, developed in molecular biology. These are implemented through the ClustalG multiple alignment program package. Alignment methods define similarity measures between character strings, which can be used to measure the similarity of two persons' daily activities, to measure change over time, or to determine the relative similarity of three or more activity diaries. The results of the research showed that both pure activities and activity-settings identified broadly the same behvioural groupings: employed workers, domestic workers, and weekend activities. The similarity of activity patterns of individuals was greater over the ten-year analysis period than the average similarity of the sample in either 1971 or 1981 . The average similarity of activity and activitysetting patterns rose from 1971 to 1981, which contradicts observations that daily routines are becoming more complex and diverse.
\end{abstract}


Empirical Research and Applications - Andrew S. Harvey and Clarke Wilson

\section{Résumé}

Les séquences d'épisodes à partir des enquêtes sur l'utilisation du temps dans un calendrier journalier sont une riche source de données sur les activités des individus et des ménages. Ces données sont utiles pour la planification urbaine. One examine ici de telles séquences, ainsi que les cites où ils ont lieu et la présence d'autres personnes, à partir de données de Halifax pour 1971 et 1981.

Des méthodes d'alignement, développées en biologie, sont adoptés à partir du logiciel Clustal G. Ces méthodes indiquent des mesures d'équivalence entre différentes séquences pour une personne ou entre personnes. Les activités et les lieux indiquent certains regroupements pour les employés payés, les travailleurs domestiques et les activités de fin de semaine. Il y a plus de ressemblance au cours des dix ans que de ressemblance d'une personne à l'autre. Contrairement à l'hypothèse de la complexification, il y a plus de ressemblance entre personnes en 1981 qu'en 1971.

Key Words: Sequences, sequence alignment, activity settings, Dimensions of Metropolitan Activity (DOMA), dynamic programming alignment, Clustal G.

\section{Introduction}

Time-use studies document the daily lives of individuals. The instruments used may be as simple as a checklist asking respondents to check those activities in which they engaged during the time being studied and/or how long they spent doing them. In contrast a time-diary records elaborate detail showing what was done, its context and how it unfolded. Typically, however, the information reported, what was done and for how long, is the same in either case. The usual reported output of diary studies is no more than what would be reported by the checklist. However, diaries are considered as providing greater accuracy. This is unfortunate on two counts. First, it is an unconscionable lack of use of the data collected. Second, a person's life is not a collection of unconnected activities to which they devote time. At any moment people are in some location, they are either alone or with someone else, and they are experiencing some emotions such as a sense of purpose, stress, satisfaction or joy. They are also coming from one set of circumstances and are moving to another. In short behaviour is manifest in a multidimensional flow of contextual dimensions that have or may not have meaning to the individual. 
Evolution of Daily Activity Patterns from 1971 to 1981:

A Study of the Halifax Activity Panel Survey

Time diaries collect information on the flow by capturing the sequence of activities and contexts. The analysis of time diary data should incorporate this richness. This paper reports an approach to capturing the context of activities reported by individuals on two days a decade apart. The findings will shed light on the extent of constancy of activities and context in people's lives and on the value of the sequence alignment approach in studying these aspects of human behaviour.

\section{Activities, Activity Settings, Activity Systems}

We know that people eat, sleep, work, travel and engage in what we can call discretionary activities. What distinguishes individuals or groups of individuals is how these fit together in their daily life pattern both in terms of order and in terms of integration with the contextual environment within which they unfold (Harvey, 1982).

\section{Activity Systems}

Context can be framed in terms of activity settings and a more activity inclusive version of behaviour settings (Barker, Louise and Ragle, 1967) that can be considered generic components of activity systems. Barker defined behaviour settings as "units of the environment that have relevance for behaviour . . . a behaviour setting coerces people and things to conform to its temporal spatial pattern." The activity system approach dates from the mid-1960s (Chapin, 1974). The elaborated approach drawing also on work by Hagerstrand (1970) and Cullen (1975) incorporates elements of motivation, constraints and perception and views behaviour in context (Harvey, 1982; Harvey, 1997). There are several dimensions of the framework within which daily activity and travel must be analyzed. These elements are actors, activities, time, and space. Ellegard (1993), drawing on the time-geographic approach of Hagerstrand, explores behaviour in terms of activity purpose, temporal flow of the day, social contact and geographic context. Her work highlights the fact that projects occur in a flow of activities and goal oriented behaviour may not be a continuous act but be intermittently undertaken as time flows on.

Actors form a group whose spatial-temporal activities are being considered. The significance of sex and employment status in determining the activity patterns of individuals is well established in the time-use/travel literature (Harvey, Elliott and Procos 1977; Robinson, Kitamura and Golob, 1992), as does the presence of young children (Chapin, 1974). Actors cannot be considered independently of other actors. Since humans are social beings, social contact exerts a strong influence on behaviour. Allardt identifies two significant dimensions of contact: loving, the individual need for attachment to other people in the immediate neighbourhood and; being, relationships between the individual and the 
community at large and the social system relating to the need for self realization, in contrast to alienation" (Gronmo and Christensen, 1982). Consequently, social contact helps shape behaviour.

Activity varies in light of the context in which it is undertaken. For example, cooking can be a work or leisure activity. Serious activity analysis will continue to suffer until appropriate activity taxonomic methods are developed. Minimally, daily time allocation can be defined in terms of (1) paid work (2) housework (3) sleep and other personal care (4) leisure. Aas (1978) characterized these as contracted time, committed time, necessary time and free time. Typically, activity schemes are much more elaborate running from about 40 to 200 activities at the collection stage. More elaborate schemes sometimes incorporate location and/or with-whom information into the activity. However, defining activities by content only is preferable. Other dimensions should be integrated in the analysis stage. The illustration in this paper uses a 15-class classification of activities reflecting both economic and social dimensions.

Time has several contextual dimensions relevant to understanding behaviour: position, the point at which actions occur (for example, weekday or weekend, morning or evening); duration, the period during which actions occur; and sequence (before or after, past, present or future). Additionally, different activities have different periodicities (Zuzanek, 1979). Harvey (1984), studying the effect of a broad range of background variables on discretionary time observed that characteristics of the day (workday, Sunday, Holiday) emerged as a major determinant of daily behaviour. This suggests a strong structuring of daily time-use by forces that fall outside the personal or household characteristics of the respondent. Research with weeklong diaries has suggested that travel is a two-stage process being determined first at the weekly level and second on a daily basis (Pas 1988). Additionally, research has shown that multiple diary days for a given respondent reduce the variance of measured behaviour (Pas, 1988). Since the analysis presented here deals only with data drawn from single day diaries, identified cross-temporal concurrence of activity could well be expected to be slight.

Space has three distinct aspects of relevance. Geographic or banal space represents arrangement and expanse - what is commonly thought of as form. Adapted space consists of sites of continual, regular or recurring activities, i.e. offices, shopping centres, parks. Channel space serves to link adapted spaces, permitting linkage of activities within the city, for example, road, rail, bike and walking routes. A space is not only a geographic location; it is a 'temporal location' as well (Lynch, 1972). This is reflected in such terms as 'daytime population' or 'bedroom community' and the work of Jannelle and Goodchild (1983) on the diurnal patterns of the city.

Behaviour depends on the three dimensions of the social environment: social circles, life space, and time or, more simply who, where and when, (Lewin, 
Evolution of Daily Activity Patterns from 1971 to 1981:

A Study of the Halifax Activity Panel Survey

1951). An individual's behaviour is a function of the roles he/she plays in each of the different social settings in which he/she participates. Behaviour deviates from the main role according to social activity setting, space, or location. For example, a father will adjust his behaviour when he is with his family, his friends, or with others and also when he is at the workplace, his home, the community, or according to the time of day or week. To properly measure the impact of social interaction on society and the behaviour of the individual, it is necessary to incorporate all the dimensions of the social environment. Each dimension finds its counterpart in time-use studies, which provide a solid basis for implementing a paradigm as shown in Table 1. The spatial and temporal activity pattern is determined by the concurrence of individual activity patterns (Kutter, 1973). These approaches are in keeping with the ideas of Moore (1963) who suggested, "Each society has a temporal structure and organization, which is a result of interaction between individuals in various settings."

Table 1

Dimensions of the Social Environment

\begin{tabular}{ccc}
\hline Dimensions & Lewin & Time-use Studies \\
\hline 1 & Social Circles & With Whom \\
2 & Life Space & Location \\
3 & Time & Time \\
\hline
\end{tabular}

Analytically it is useful to define units of the social environment such that they are amenable to analysis. At each moment in time individuals exist in a certain social circle and life space. Ideally one would divide social circles into at least three circles: family, friends, and other acquaintances. However, data problems required the reduction of the social circles to two: the family circle and a circle of other acquaintances (Harvey and Taylor, 2000). Social space can be defined in terms of household space, workplace or school related locations, community space, non-work related social/recreational space and houses of friends and relatives, etc. (Harvey and Taylor, 2000). However, the segregation of the social circles is not easily accomplished since in real life they intermingle, at times becoming a single entity, which we call society. Schneider (1972) had difficulties isolating the social actors in separate social settings. 
In spite of the difficulties, it is possible to operationalize social environment for analysis purposes (see Table 2). Here we draw on the work of Harvey and Taylor (2000). Social circles were created using with-whom data in the timeuse surveys. Time spent sleeping was deducted since for social interaction one is only concerned with the time that people spend awake. Analysis was carried out to determine levels of multiple interactions and avoid the duplication of time. It was found that multiple interaction was not statistically significant and it became evident that the division between friends and acquaintances was not reliable. Aggregation resulted in three types of interaction that were integrated with the social space to create the two dimensional social environment, social circle and social space.

Table 2

Two Dimensional Social Environment

\begin{tabular}{ccccc}
\hline Social Circle & Household & Workplace & Community & Transit \\
\hline Alone awake & $\begin{array}{c}\text { Alone at } \\
\text { home }\end{array}$ & $\begin{array}{c}\text { Alone at } \\
\text { workplace }\end{array}$ & $\begin{array}{c}\text { Alone in } \\
\text { community }\end{array}$ & $\begin{array}{c}\text { Alone in } \\
\text { transit }\end{array}$ \\
With family & $\begin{array}{c}\text { With family } \\
\text { at home }\end{array}$ & $\begin{array}{c}\text { With family } \\
\text { at workplace }\end{array}$ & $\begin{array}{c}\text { With family } \\
\text { in community }\end{array}$ & $\begin{array}{c}\text { With family } \\
\text { in transit }\end{array}$ \\
$\begin{array}{c}\text { With others } \\
\& \text { multiple }\end{array}$ & $\begin{array}{c}\text { With others } \\
\text { \& multiple at } \\
\text { home }\end{array}$ & $\begin{array}{c}\text { With others } \\
\text { \& multiple at } \\
\text { workplace }\end{array}$ & $\begin{array}{c}\text { With others } \\
\text { \& multiple in } \\
\text { community }\end{array}$ & $\begin{array}{c}\text { With others } \\
\text { \& multiple in } \\
\text { transit }\end{array}$ \\
\hline
\end{tabular}

\section{Data}

Time diaries, as suggested above, provide the most complete and accurate means of accounting of time-use and elucidating individual behaviour. A time diary captures all activities over a recording period typically of 24 to 48 hours and sometimes running up to one week. By its nature, it permits and facilitates the recording of a number of contextual dimensions, including where and withwhom activities are done, attendant with each particular act. The several contextual dimensions mutually aid recall and enhance data accuracy. 
Evolution of Daily Activity Patterns from 1971 to 1981:

A Study of the Halifax Activity Panel Survey

Panel studies obtain data on the same subjects at two or more points in time. While they are not uncommon, there have been very few time-use panel studies. Michelson (1972) collected time-use data as part of a three-wave panel over 18 months for a very specialized sample of movers. In the 1970's and 1980's, Juster and colleagues conducted a sort of double, short term-long term panel. They interviewed respondents in four waves over a year spanning 1975-76 and conducted a follow-up in four waves in 1981-82 (Juster, 1985). More recently the German Socio-Economic Panel study collected time-use data annually over an extended period of time (Merz and Rauberger, 1992). However, they used stylized questions, rather than a diary, to capture time allocation.

This study draws on data from the Halifax, Nova Scotia time budget panel study. The effective Halifax panel consists of 453 persons who completed time diaries in the fall of 1971 and in the fall of 1981. It is a subset of the sample used in the Dimensions of Metropolitan Activity (DOMA) study carried out in 1971 (Harvey and Elliott, 1983). In essence, if the respondent completed a diary for a Tuesday in November in 1971, they later completed a diary for a Tuesday in 1981. The Halifax panel remains, to the best knowledge of the authors, the longest time period covered by a time-use panel. A sample of 75 persons was drawn from the main panel to illustrate the activity patterns that emerge using sequence alignment methods. Table 3 shows their time budgets for 1971 and 1981 using 15 activity categories.

Analysis of the main panel (Harvey and Elliott, 1983) showed that significant declines were registered for home chores, laundry, child-care, sleep, leisure travel, other work, personal care and entertainment. Significant gains were registered for television viewing, other leisure, other house care, reading books, personal travel, shopping, resting and reading the paper. Declines in home chores and leisure travel were just offset by increases in marketing and other house related activities and by personal travel that includes travel for shopping and to accompany children.

Examined in relation to DOMA random samples studied in 1971 and 1981, panel declines in sleep, leisure travel and personal care appear to have occurred due to the aging process since there was no significant change for the random sample. Similarly, increased time allocations to other leisure, other home care, shopping, resting and reading the paper were only significant for the Halifax panel. In contrast, eating and magazine reading declined significantly for the DOMA random sample but not for the Halifax panel; while study, errands, other childcare and conversation increased significantly for the random sample but not for the panel (Harvey and Elliott, 1983). 
Table 3

Time Budgets of the Halifax Panel Respondents

(75- person sample)

\begin{tabular}{|c|c|c|}
\hline \multirow{2}{*}{ Activity } & \multicolumn{2}{|c|}{ Time in minutes } \\
\hline & 1971 & 1981 \\
\hline Sleep, rest & 475 & 494 \\
\hline Personal care & 65 & 49 \\
\hline Eating & 94 & 94 \\
\hline Paid work & 223 & 205 \\
\hline Domestic work & 74 & 79 \\
\hline Cooking, dish washing & 63 & 50 \\
\hline Family care & 28 & 31 \\
\hline \multicolumn{3}{|l|}{ Shopping, use of services } \\
\hline \multicolumn{3}{|l|}{ Volunteer, community } \\
\hline Activity & 15 & 16 \\
\hline Sport, hobbies & 42 & 53 \\
\hline Entertaining, socializing & 75 & 44 \\
\hline Education, study & 20 & 0 \\
\hline $\mathrm{TV}$, media, reading & 155 & 223 \\
\hline Travel & 74 & 74 \\
\hline Unknown & 6 & 6 \\
\hline Total & 1,440 & 1,440 \\
\hline
\end{tabular}

Of particular note with respect to activity sequencing, which is the focus of the current analysis, is the significant decline in sleeping and the significant increase in resting. The panel report posed the question "Does regular sleep get replaced by episodes of resting [sleep] as one ages?" (Harvey and Elliott, 1983)

The foregoing results focus on activity content independent of context. However, behaviour ultimately cannot be considered devoid of context. Minimally, location, social contact and time are major contextual elements structuring behaviour (Harvey, 1982). Ideally one would also know something of the subjective environment as well.

In evaluating quality-of-life "the degree of fit or congruence between an event and its context ...is of key concern." (Goodhart and Zautra, 1984:259) It is argued that responses to and the outcomes of life events are a function of the 
Evolution of Daily Activity Patterns from 1971 to 1981:

A Study of the Halifax Activity Panel Survey

degree of congruence between the events and other events deemed normative by psychological and social contextual standards.

\section{Alignment Analysis of Activity Data}

\section{Introduction}

Previous work (Wilson, 1998a, 1998b) has shown the value of using sequence alignment methods developed in mathematics and biology for studying individual behaviour drawn from time diaries. Alignment analysis (also called optimal matching) can provide an ideal means of identifying and defining daily patterns and for evaluating daily behaviour of individuals and groups of individuals. Much of the value of such an approach will be in its ability to identify cultural constants and life structuring behaviours. Such knowledge can have many applications including quality-of-life (QOL) assessment.

Goodhart and Zautra (1984) argue for an ecological approach to QOL assessment. Such an approach, they argue, requires assessment of the events of the person, an assessment of the social and psychological contexts and measurement of the fit between the two. In their view, measures of eventcontext congruence are indicators of QOL. There is a need to define norms both subjectively and objectively. Goodhart and Zautra suggest one could derive socially normative life events empirically. Diary data provide an optimal vehicle for doing so with respect to daily behaviour and sequence analysis is a valuable tool for identifying socially normative life events empirically.

At the same time that we seek to define cultural constants (and in the face of such constants), we can ask: Is there a separate space for individuals? Individuals can be distinguished with fair certainty by biological features, fingerprints, DNA, and more recently by eye structure. Can individuals also be identified by behaviour patterns? Perpetrators of certain deviant behaviour are often identified by their MO (modus operandi). May that not be true of normal behaviour as well?

One way of examining these questions is by examining the behaviour of individuals with a view to identifying normative behaviour and the extent to which such behaviour, albeit normative, plays out differently in individual lives. Time diaries for two days a decade apart from a sample of individuals in a Halifax Canada panel provide a perfect opportunity to examine these questions.

To define a norm is to define a meaningful grouping of similar behaviours. There have been several sustained efforts to define activity patterns in terms of various elements of the social spaces and social choices described above (see Pas, 1983; Koppelman and Pas, 1984; Golob, 1984; Recker et al, 1985). However, no method has been widely adopted as a standard and, consequently, 
none has replaced time budget comparisons as the basis for describing activity patterns.

The analysis of organic molecules, particularly proteins and nucleic acids addresses questions that are analogous to those addressed here. Questions relating to biological implications of protein and nucleotide sequences and the roles of specific subsequences have analogues in the activity system perspective on human behaviour. We may ask whether two groups' activity patterns are similar, whether a person's pattern is more similar to a second person's than to another, and how much patterns change over time. We may also be interested in identifying key subpatterns that are common among people but which do not exactly match for any.

Biologists have used character sequences to represent molecular structure for decades. Before 1970, the primary analytical tool available was dot matrix analysis, which is a qualitative examination of matrices that show the matching pattern of elements in all positions of a pair of sequences. Since 1970 there has been an explosion of algorithms for calculating a variety of similarity or distance measures for pairs of molecules.

\section{Dot Matrix Analysis of Character Sequences}

Any pair of character sequences can be written as the row and column headings of a matrix and dots or stars are placed in cells in which the row and column elements match. Figure 1 shows a matrix analysis of a sequence identity [london \} and of two similar sequences, [montreal] and [montebello].

When the two sequences are identical, the table is square and the dot pattern fills the main diagonal. In addition any repetition of characters in the sequences generate off-diagonal dots. This is shown in the [london] table, where there are two stars in the upper right and lower left. All other stars are on the main diagonal.

The sequences [montreal] and [montebello] are intuitively similar but to some extent are different. Their matrix analysis also shows a strong but not prefect diagonal pattern, and more non-diagonal elements. Similarly, if we were to analyse [regina] and [winnipeg], we would see a number of matches but no linear pattern. While dot-matrices can reveal much about similarity of sequences, they are unwieldy when more than a few are involved. 
Evolution of Daily Activity Patterns from 1971 to 1981:

A Study of the Halifax Activity Panel Survey

\section{Figure 1}

Dot Matrix Analysis of Character Sequences 


\section{Dynamic Programming Alignment Algorithms}

The process of calculating the pair-wise similarity measure defines the best pattern of matches between elements of the two sequences, which are illustrated by writing them one above the other. The best match is defined as the maximum matching score (or the minimum conversion cost) that can be achieved by converting one sequence into the other using only eligible operations. Eligible operations are identical matches, substitutions (or inexact matches), insertion of an element from one sequence into the other, and conversely deletion of an element. Gaps are inserted in either sequence as necessary to accommodate insertions and deletions to align matching characters. Insertions and deletions are descriptions of the same operation from the perspective of one or other sequence. They always occur in pairs and are usually called indels. An example of one possible alignment of [montreal] and [montebello] is illustrated below.

montre-al--

mont-ebello

The quantification of matrix methods involves developing rules for counting and scoring matches so that scores accumulate. More matches give higher similarity scores or lower conversion costs. Algorithms have been developed for various applications. Optimality of alignment is defined in the sense that no alternative arrangement of matching characters and inserted blanks can give a higher similarity (or lower distance) score than the one found.

Let two sequences of $n$ and $m$ letters (or words) from the alphabet or vocabulary $\mathbf{A}$ be denoted by $\mathbf{a}$ and $\mathbf{b}$. Elements of $\mathbf{a}$ and $\mathbf{b}$ are $a_{i}, i=1 \ldots n$ and $b_{j}, j=1 \ldots m$. There is a similarity matrix $\mathbf{s}(a, b)$ defined on the alphabet such that $\mathbf{s}(a, a)>0$. The values of $\mathbf{s}(\mathrm{a}, \mathrm{b}), \mathrm{a}<\mathrm{b}$, may be greater or less than zero but are less than $\mathbf{s}(\mathrm{a}, \mathrm{a})$ and $\mathbf{s}(\mathrm{b}, \mathrm{b})$.

Let the similarity score of two elements at position $(i, j)$ be $\mathrm{s}\left(a_{i}, b_{j}\right)$ or more simply, $\mathrm{s}(i, j)$. This is read from the matrix or is determined by the indel penalty. Indels are penalized by a constant penalty, as:

$$
\mathrm{s}(i,-)=\mathrm{s}(-, j)=-c
$$

We initialize the process by defining an element 0 that is prefixed to each sequence and for which

$$
\mathrm{s}(i, 0)=-i c \text { and } \mathrm{s}(0, j)=-j c
$$

Let the similarity measure of $\mathbf{a}$ with $\mathbf{b}$ from their beginning to any position $(i, j)$ be $\mathrm{S}(i, j)$ and the similarity for the complete alignment be $\mathrm{S}(a, b)=\mathrm{S}\left(a_{i}, b_{j}\right)$, where $i=n$ and $j=m$. 
Evolution of Daily Activity Patterns from 1971 to 1981:

A Study of the Halifax Activity Panel Survey

The matrix $\mathrm{S}(i, j)$ is filled recursively from the top left position $(0,0)$ to the bottom right position $(n, m)$. Any optimal path ending at element $(i, j)$ is composed of an optimal path ending at a previous position plus the value of the highest similarity score at position $(i, j)$. The key to understanding the algorithm is the observation that any alignment must end in one of three ways:

$$
\left\langle\begin{array}{l}
a_{i} \\
b_{j}
\end{array}\right\rangle \text { or } \quad\left\langle\begin{array}{c}
a_{i} \\
-
\end{array}\right\rangle \text { or } \quad\left\langle\begin{array}{l}
- \\
b_{j}
\end{array}\right\rangle
$$

This means that the end of an alignment at $(i, j)$ must be:

- $\quad$ a match of the $i$-th character of $\mathbf{a}$ with the $j$-th character of $\mathbf{b}$;

- insertion of the $i$-th character of $\mathbf{a}$ into $\mathbf{b}$ creating a gap in $\mathbf{b}$; or

- insertion of the $j$-th character of $\mathbf{b}$ into $\mathbf{a}$ creating a gap in $\mathbf{a}$

Then any optimal score $\mathrm{S}(i, j)$ can be found by finding the maximum of three sums:

$$
\mathrm{S}(i, j)=\max [\mathrm{S}(i-1, j-1)+\mathrm{s}(i, j), \mathrm{S}(i-1, j)+\mathrm{s}(i,-), \mathrm{S}(i, j-1)+\mathrm{s}(-, j)]
$$

The optimal similarity value of each cell $(i, j)$ requires a search of only three previous cells: the one above, the one to the left of, and the one diagonally above to the left of $(i, j)$. The program records a pointer at each cell indicating the previous optimum cell. The path backward from cell $(n, m)$ to $(0,0)$ is found by following these pointers.

Needleman and Wunsch (1970) published the first algorithm to evaluate sequence similarity quantitatively. The first text on alignment methods covering many disciplines was that of Sankoff and Kruskal (1983). Waterman (1995) gives a more up to date account of applications and algorithms related to biology.

Pair-wise alignment can be generalized to multiple alignments of $\mathrm{N}$ sequences by constructing comparison tables in $\mathrm{N}$ dimensions and extending equation (3) as required. However, the time demands on computers of multiple alignments increase explosively as the number of sequences increases, becoming unmanageable after about 10 sequences of moderate length. Multiple alignments are therefore implemented using some type of approximation method based on data from pair-wise alignments. This is the strategy followed by ClustalG (see below).

When whole sequences are examined the method is called global alignment. Subsequences may also be examined using local alignment algorithms. These tend to be more complex and demanding of computer facilities than global alignment. 
The methods as developed to date are mainly unidimensional, reflecting the kinds of problems addressed by developers of algorithms. This presents a limitation for applications in social science where multiple dimensions are typical when considering event sequences. Joh, Arentz and Timmermans (2000) have extended the algorithms to handle multiple sequences of elements for each individual. For example, person (i) could record separate sequences of activity and location. A sample of size $n$ would consist of $2 n$ sequences that are aligned in pairs by an amended multiple alignment algorithm. An alternative is to embed the additional data dimensions that define the activity setting in the coding system. The ClustalG amendments to ClustalW and ClustalX used in molecular biology permit this treatment of activity settings and the more complex coding system that it requires.

\section{ClustalG}

The Clustal family of alignment software originated in work by Higgins and Sharp (1988) and has been extensively developed at the European Molecular Biology Laboratory (Thompson, Higgins and Gibson, 1994). It is difficult to find an optimal path through a multidimensional comparison table or alternatively to find the maximum similarity score using an $n$-dimensional extension of equation (3) because the search space is vast. An exercise in Durbin et al (1998) shows that to implement equation (3) for about 15 sequences of 1000 elements would take the lifetime of the sun. The strategy used by all multiple alignment programs that offer solutions for more than about a dozen sequences is to employ some heuristic strategy to search the space defined by an $n$-dimensional comparison table.

The Clustal programs base their approach on defining a tree that progressively combines pairs of sequences or sequence groups on the basis of distance measures. Durbin et al. (1998) give a summary of the process followed by a package called ClustalW. Some specifically biological features were eliminated in the preparation of ClustalG, but the process of moving from pairwise similarity scores to distance measures to a multiple alignment remains the same. ClustalG writes a file of similarity scores and the percentage of identical elements for all pair-wise alignments to a file. This file may be passed to other software packages and used to analyse similarity statistics.

ClustalG has no limit on the number of sequences that may be input, but the time required for calculation of pair-wise similarities and the compilation of the guide tree increases rapidly with the number of sequences analysed. This puts rather stringent limits on the number of sequences examined. One of the authors of this paper has illustrated ClustalG with 248 diaries (Wilson, 2001). A group at the University of Eindhoeven have tested alignment methods against alternatives using over 1000 travel diaries. The amount of calculation involved 
Evolution of Daily Activity Patterns from 1971 to 1981:

A Study of the Halifax Activity Panel Survey

is huge, but computational capacity is growing rapidly. It would seem that essentially complete analyses of many research data sets of the kind used by transportation planners, geographers, demographers and many other disciplines are possible. Analysis of national diary surveys of time use that have recorded anywhere from 5000 to 50,000 diaries remains a problem.

The key difference between ClustalG and the rest of the Clustal family of software is that ClustalG represents elements of sequences by words of up to six letters rather than by single letters. Letter positions in the word convey the dimensions of information about the sequence, in this case the settings in which activities occur. The six-character word can accommodate over 300 million categories of information about an event, for the present eliminating limits on the complexity of coding schemes for sequential events.

\section{Alignment Analysis of the Halifax Panel Data}

\section{Behavioural Sequences}

A random sample of 75 respondents (150 diaries) from the Halifax panel survey is used to describe activity patterns in general, and to examine the relative stability of individual and population patterns over a ten-year period. Table 4 shows the respondent characteristics. The average age was ten years more in 1981 than in 1971. The distribution of marital status did not change over the ten years. The age, sex and marital status of the sample used in this analysis were within 3 percent of the values for the panel as a whole.

Table 4

Respondent Characteristics, 1971

\begin{tabular}{lc}
\hline Characteristic & Percent \\
Sex: & \\
Male & 45 \\
Female & 55 \\
& \\
Age & 37 years \\
Marital Status: & \\
Married & 82 \\
Single & 18 \\
& \\
\hline
\end{tabular}


Two sets of sequences were created for this study. One is composed of activity episodes and the other from activities augmented with data on where an activity occurred and who else was present. We call the former activity sequences and the latter activity-setting sequences. We take the word setting to be synonymous with context. Activities are represented by two-letter elements. Activity-settings are represented by four-letter elements, of which the activity occupies the first two positions. The location and accompaniment conditions are represented by single letters and occupy, respectively, positions three and four in the setting elements. They do not appear in the activity sequences. The coding schemes are shown in Tables 5 and 6. An upper-case letter is used in position one of multiletter elements to aid readability.

\section{Table 5}

\section{Activity Sequence Code Key}

\begin{tabular}{|c|c|c|c|}
\hline Activities & Letter & Activities & Letter \\
\hline Work & Wk & Education & li \\
\hline Cleaning, food preparation & $\mathrm{Ck}$ & $\begin{array}{l}\text { Volunteer, community } \\
\text { Activity }\end{array}$ & $\mathrm{Vc}$ \\
\hline Other domestic & $\mathrm{Dm}$ & Personal care & $\mathrm{Pc}$ \\
\hline Family care & $\mathrm{Fc}$ & Sleep, rest & $\mathrm{Zz}$ \\
\hline Travel & $\operatorname{Tr}$ & Entertainment, visiting & Fn \\
\hline Shopping, services & $\mathrm{Sh}$ & Hobbies, sport, leisure & $\mathrm{Hs}$ \\
\hline Eating & Et & $\begin{array}{l}\text { Media } \\
\text { (TV, radio, reading) } \\
\text { Not stated }\end{array}$ & $\begin{array}{l}\mathrm{Tv} \\
\mathrm{Xx}\end{array}$ \\
\hline
\end{tabular}

Each activity and context sequence was created by recording episodes as 30minute time blocks. For example, a two-hour domestic work episode would generate four domestic work events. Episodes are rounded upward so as not to lose short activities.

Trees are graphs that may be used to model matrices of measures of proximity (or distance) among a set of objects, in this case activity diaries. As simple illustrations of proximity data, they may be drawn without an origin or orientation in an unrooted form. If some hypothesis or evidence is available about the evolution or development of the object set, as is the case when measures of similarity of animal species are used, a parent node or root may be 
Evolution of Daily Activity Patterns from 1971 to 1981:

A Study of the Halifax Activity Panel Survey

defined that represents a most general state or class, from which all objects and groups of objects descend. As no evolutionary or developmental process is hypothesized here, no root has been illustrated.

\section{Table 6}

Activity Setting Code Keys

\begin{tabular}{lclc}
\hline \multicolumn{1}{c}{ Locations } & Letter & \multicolumn{1}{c}{ Persons Present } & Letter \\
& & & \\
Home & $\mathrm{H}$ & Alone & $\mathrm{a}$ \\
Work & $\mathrm{W}$ & $\begin{array}{l}\text { Family } \\
\text { (and other persons) }\end{array}$ & $\mathrm{f}$ \\
Other & $\mathrm{O}$ & $\begin{array}{l}\text { Other persons not family } \\
\text { Not stated }\end{array}$ & $\mathrm{p}$ \\
& & Not stated & $\mathrm{x}$ \\
\hline
\end{tabular}

Figures 2 and 3 are unrooted trees illustrating the proximities of the 150 diaries. Proximity is based on ClustalG pair-wise similarity scores. The first figure shows activity sequences and the second shows activity-setting sequences. Each tree corresponds to an alignment of 150 sequences ( 75 diaries for 1971 and 75 for 1981).

The whole alignments are 150 rows long and, for activity-settings, may be four pages wide or more, depending on the assumptions made about substitution and indel penalties. Because of their size, we have reproduced only samples of the parts of the alignments that relate to different sectors of the trees and which describe the activities of a group. The sample alignments have been printed in two blocks so that each individual's day wraps onto two lines. Even with the wrapping, the blocks had to be trimmed at the beginning and end of the day to remove repetitive sleep episodes and leading and trailing gaps that are generated by differing sequence lengths.

Alignments used a score of ten for matches, and penalties of eight and three for the first and for successive gaps created by insertions and deletions. Partial scores were given for substitutions among a set of work-oriented activities (employment, domestic work, family care, cooking) and among personal care activities (rest, eating, personal care). Activity-setting alignments also gave partial scores for matches of location or persons present when activity substitutions occurred. The matching and substitutions scores are the elements of $\mathrm{s}(i, j)$ of equation (3). The gap penalties are the $\mathrm{s}(i,-)$ and $\mathrm{s}(-, j)$ terms, also of equation (3). 
Empirical Research and Applications - Andrew S. Harvey and Clarke Wilson

The patterns in the alignments relate to the major divisions of the trees and were identified by eye. The trees are interpreted as illustrating the major behavioural groups of the panel sample, and hence of the Halifax population. The tree branch labels and the alignment lines contain a respondent number, the year, and the day of the diary. References to clock times are useful in describing positions in the trees.

\section{Activity Sequences}

The first tree (Figure 2) shows behavioural groupings based on activity sequences. It displays, roughly, three collections of members. The largest, which we call Pattern A, occupies the bottom of the diagram from about 3:00 o'clock to 9:00 o'clock. The top of the diagram can be divided into two groups occupying the positions from about 9:00 to 12:30 (Pattern B) and from 12:30 to 3:00 (Pattern C).

\section{Pattern A: Employed Workers}

Pattern A consists of 76 diaries of persons who work for pay for the majority of the day. Morning and noon meals occur fairly regularly as required by the time demands of paid employment. In five of the six diaries shown, workers do a shopping errand either on the way to work or after work. This is naturally not uncommon but is not strictly representative of the Pattern A people as a whole. The group shown does less domestic work than other group members. As a whole, the group engages in comparatively little socializing, hobbies or recreation, or television viewing. Television is the most common non-work activity.

\begin{tabular}{|c|c|}
\hline $25477 \mathrm{th}$ & $\begin{array}{l}\text { Z zPCFCEtPCTVTVTrXxWkWkWkWkWkWkWkWkWkWkXxTrShTr } \\
\text { EtTrWk------WkWkWkWkWkWkWk }\end{array}$ \\
\hline $34378 \mathrm{mn}$ & $\begin{array}{l}\text { ZzZzZzZzZzPcCkEtWkWkTrFcWkWkWkWkWkWkWkTrShTrEt } \\
\text { WkWk-----WkWkWkWkTrWkTr }\end{array}$ \\
\hline $137 \mathrm{th}$ & $\begin{array}{l}\text { ZzZzZzZzPcDmCkEtTrXxWkWkWkWkWkWkWkWkWkWkTrShTr } \\
\text { EtWkWk-----WkWkWkWkTrTrSh }\end{array}$ \\
\hline $24578 w d$ & $\begin{array}{l}\text { ZzZzZzZzZzPCEtEtTvTrXxXxWkWkWkWkWkWkWkEtEt---- } \\
\text {--WkTrShTVTrTrSh }\end{array}$ \\
\hline $31907 \mathrm{wd}$ & $\begin{array}{l}\text { ZzZzZzZzPCCkPCEtTrXxWk--WkWkWkWkWkWkWkWkEtWkWk } \\
\text { WkWkWk------WkWkWkWkWkWkWk }\end{array}$ \\
\hline $20268 \mathrm{fr}$ & $\begin{array}{l}\text { PcFCTrTrTrTrXxWkWkWkWk-WkWkWkWkWkWkWkWk------ } \\
\text { WkWkWkWkWkWkWk }\end{array}$ \\
\hline
\end{tabular}


Evolution of Daily Activity Patterns from 1971 to 1981:

A Study of the Halifax Activity Panel Survey

Figure 2

Activity-Sequence Similarity Tree 
Empirical Research and Applications - Andrew S. Harvey and Clarke Wilson

\begin{tabular}{|c|c|}
\hline $34378 \mathrm{mn}$ & WkWkTrEtTvTvTrShShShShTrTvTvTVTvPcZzZz---- \\
\hline $137 \mathrm{th}$ & ShShTrEtTrShTrShShTrPcTvTvTvPCTvZzZz------ \\
\hline $4578 w d$ & ShShShShTrCkEtPcPcPcPCPCPCPC--- \\
\hline $31907 w d$ & WkWkWkWkWkWkTrPcEtEtTVTvTvTV----- \\
\hline $0268 \mathrm{fr}$ & $\begin{array}{l}\text { WkWkWkWkWkXxTrPcEtEtCkTvFcFCFCCkCkCkPcPcTr } \\
\text { FnFn }\end{array}$ \\
\hline
\end{tabular}

Pattern B: Homemakers

The 36 people of Pattern B have apparently endless free time that is devoted to watching television, socializing, and to hobbies or sport. Five persons worked for pay. Twenty-four of the Pattern B diaries were weekend diaries. They also record considerable shopping activity.

\begin{tabular}{|c|c|}
\hline 4577 fr & $\begin{array}{l}\text { ZzZzZzZzTvPcCkTvTvTvTvTvCkCkTvTvTvTvTvZzZzPC } \\
\text { PcEtEtTVTVTVTrWkWkWkWkWkWkWk }\end{array}$ \\
\hline $34338 t u$ & $\begin{array}{l}\text { ZzZzZzCkCkCkCkDmDmTrTrShShShShShTrTrCkHsHsHs } \\
\text { HsHsTVTVTVTVTvCkCkEtEtCkTvTv }\end{array}$ \\
\hline $38867 \mathrm{sn}$ & $\begin{array}{l}\text { ZzZzZzPcCkFcEtTrFnFnTrHsHsTrFcTrPcTvTvTrZzTr } \\
\text { CkCkTvEtTvTVTvCkCk-------Tv }\end{array}$ \\
\hline 2658 th & $\begin{array}{l}\text { ZzPcPcDmCkFcTvCkEtEtCkTvTvPcPcTrShShTrCkzzZz } \\
\text { CkEtFnFnCkTvTvCkCkCkCkPcEtTv }\end{array}$ \\
\hline $12338 \mathrm{sn}$ & $\begin{array}{l}\text { ZzZzZzCkTvTvTvTvTvTvTvCkCkEtTvTvTvTvTvPcPc } \\
\text { CkTVTvTVTVTVTVTVTVCkTvTvCkEtCk }\end{array}$ \\
\hline $2518 \mathrm{sn}$ & $\begin{array}{l}\text { ZzZzPcHsPcPcCkEtTvTvCkDmDmDmTvTrTrFcFcTrTrTr } \\
\text { DmTvTvTvTvTvTvTvTvTvTvCkEtCk }\end{array}$ \\
\hline $4577 \mathrm{fr}$ & 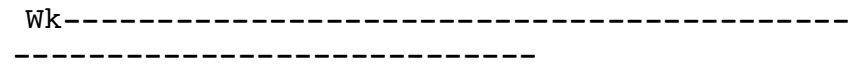 \\
\hline $34338 t u$ & TvTvTvTvTvTvTVTVTVCkEt---- \\
\hline $38867 \mathrm{sn}$ & $\begin{array}{l}\text { EtCkCkPcZzZzZz----ZzZzZzZzZzPcCkTvFCTvTvDm } \\
\text { PCZzZz-- }\end{array}$ \\
\hline 2658 th & TvCkDmPcTvTVTVTVTvZzZzZzZzZz----------- \\
\hline $12338 \mathrm{sn}$ & ----TVTVTVTVTVTVPcZzZzZzZzZzZzZz----------- \\
\hline $8 \mathrm{~s}$ & WkWkTvTVTVTVTVTvPcZzZzZzZz \\
\hline
\end{tabular}

Pattern C: Week-enders

Pattern $\mathrm{C}$ people do much more domestic work than the other groups, although the diaries show somewhat more than the group as a whole. The group does not shop as much as Pattern B and undertakes more family care than either of the 
Evolution of Daily Activity Patterns from 1971 to 1981:

A Study of the Halifax Activity Panel Survey

other groups. A subgroup of Pattern C people watch considerably more television than the rest, and tend to socialize. The mealtimes of this homemaking group is more diffuse than for Patterns A and B. Domestic work occurs generally during the middle of the day but is nowhere nearly as regular as paid work.

\begin{tabular}{|c|c|}
\hline $2657 \mathrm{th}$ & $\begin{array}{l}\text { DmDmFcTvPcTrFcFcTrShShShShTrPcTrFcTrPcCkEtFcFc } \\
\text { DmDmDmCkCkDmCk----EtEtTvFc }\end{array}$ \\
\hline $36267 \mathrm{sn}$ & $\begin{array}{l}\text { ZzZzZzZzZzZzPcCkEtTvFcPcDmTrShShShTrDmCkEtEt } \\
\text { DmDmDmDmCkCkTvTvCkFnEtEtEtCk }\end{array}$ \\
\hline $5698 \mathrm{sa}$ & $\begin{array}{l}\text { ZzZzZzZzZzZzZzPcEtDmDmDmDmDmDmDmDmDmDmEtEtDm } \\
\text { DmDmDmDmDmDmDmEtEtTvTvTvTvTv }\end{array}$ \\
\hline $34718 w d$ & $\begin{array}{l}\text { ZzZ zEtCkDmDmTvFcF cF cF cDmDmDmDmDmDmDmDmEtDmDm } \\
\text { DmDmDmDmDmDmDmDmDmDmDmDmDmDm }\end{array}$ \\
\hline $23277 \mathrm{tu}$ & $\begin{array}{l}\text { ZzZzZzZzPcCkEtEtEtPcDmPcDmEtDmCkDmDmDmTvTv } \\
\text { CkEtCkDmDmDmDmDmDmEtDmDmDmTvTv }\end{array}$ \\
\hline $9428 m n$ & $\begin{array}{l}\text { ZzZzZzZzZzTvTvCkEtCkDmDmDmDmEtDmDmDmCkEtEt } \\
\text { DmDmDmDmTvDmDmDmDmDmCkPcEtEtEt }\end{array}$ \\
\hline $2657 \mathrm{th}$ & CkTvFcCkTvTvTvPcTrFnFnFnTrPcCkTvTvTv------- \\
\hline $36267 \mathrm{sn}$ & 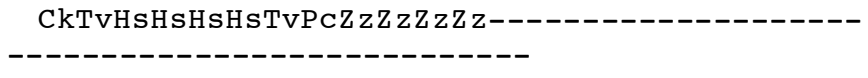 \\
\hline $5698 \mathrm{sa}$ & 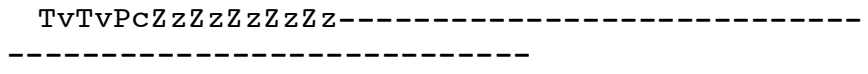 \\
\hline $34718 w d$ & 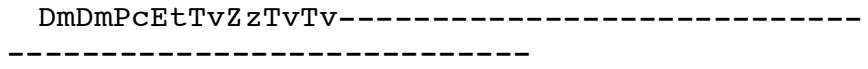 \\
\hline $23277 t u$ & 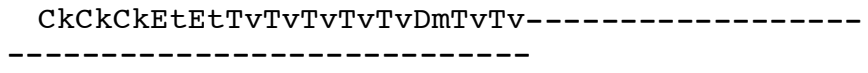 \\
\hline $9428 \mathrm{mn}$ & CkPcTrFnFnFnFnFnFnFnTrCkEtZzZzZz----------- \\
\hline
\end{tabular}

\section{Activity-setting Sequences}

Figure 3 shows the activity-setting diaries. The tree shows four lobes of branches indicating four behavioural groups (Patterns D through $G$ - The codes forming these patterns are given in the Appendix for lack of space here). Pattern D occupies the bottom of the tree from about 3:00 o'clock to about 7:30. Pattern E extends from 7:30 to about 11:00 o'clock. Pattern E occurs from 11:00 to 12:00 and Pattern F from about 12:00 to 3:00. 
Empirical Research and Applications - Andrew S. Harvey and Clarke Wilson

Figure 3

Activity-Context Similarity Tree 
Evolution of Daily Activity Patterns from 1971 to 1981:

A Study of the Halifax Activity Panel Survey

Pattern D - Employed workers

The patterns of workers are, again, the most distinctive. Pattern D consists of 63 diaries, most of which show a large block of work activity during the middle of the day. Workers have comparatively regular meal times, especially in the morning and at midday. Evening meals are more dispersed. Naturally, travel is common before and after work. Pattern D contained 385 travel elements, or 6.1 per person. These are not episodes, because they have been constructed as 30minute blocks, but they overestimate total activity time as well, because block counts are rounded upwards. The element count simply measures activity time relative to other patterns in the alignment. Shopping is fairly uncommon with only 1.3 elements recorded per person.

Pattern D consists of two blocks of diaries that seem to be differentiated by whether the work activity occurs alone or with other people. This may indicate an important difference in working conditions, or it may be a coding artefact created by weak instructions regarding how the presence of other persons should be interpreted.

Pattern E - Socializers

Pattern E consists of 31 diaries that display a mixture of domestic work, including family care, free time activity, including television viewing, socializing, and hobbies, and education. Twenty of the diaries were weekend days, which accounts in part for the mix of activities. Domestic and education activity tend to occupy the morning and early afternoon, with free time activity tending to occur later in the day. The Pattern E group recorded 6.7 travel elements and 2.4 shopping elements per person. Socializing, education, and shopping are out-of-home activities and often occur with non-family people. This indicates that Pattern E people are highly mobile and active.

Pattern F - Residual

The 18 diaries of Pattern F, which were the last to be added to the whole grouping, contained 11 weekend days. They are residual diaries that did not match well with themselves or with other groups. Pattern $\mathrm{F}$ activities are a mixture of paid work, domestic activity, and free time. Respondents recorded the most travel (7.1 elements per person) and the second-most shopping (1.9 elements per person).

A visual inspection of the whole alignment indicates that Pattern F people tend to be alone less than most other persons.

\section{Pattern G - Domestic}

The 38 diaries of Pattern G contain the longest organized episodes of domestic work and family care. Pattern $G$ respondents travelled and shopped less than any of the other groups, with 2.6 travel elements and 0.7 shopping elements. They spent more time alone than members of other groups but a large majority 
reported at least some activity with family members indicating that these people did not live alone. Meal times are slightly more regular than in Pattern E and F, but are less regular than employed workers.

The domestic and family care activities are somewhat analogous to work activity of Pattern D, occupying most of the morning and afternoon. However, domestic activity is interspersed with episodes of free time (usually television, hobbies, or socializing) much more than work activity.

\section{Similarity of Behaviour from 1971 to 1981}

One objective of this study is to discuss the stability or variability of behaviour as reflected in activities and activity contexts over the 1971-1981 time period. Table 7 shows the average percent identity scores generated by the ClustalG for the activity and activity-setting sequences. The percentage of identical elements in a pair-wise alignment is a generalization of the similarity score that standardizes for the difference in sequence length. In each year there are 2775 pairwise similarity comparisons possible among 75 respondents. Restricting the comparisons to the same individual's diaries in 1971 and 1981, there are 75 scores.

The 1971 and 1981 scores show a consistent increase in the similarity of behaviour over the decade in terms of activity and context sequences. Average activity similarity increased by 2.5 percent while context similarity increased by over 25 percent. Expressed the other way around, diversity of behaviours, especially behaviour in context, declined during the ten-year period.

The similarity of activity patterns of individuals between 1971 and 1981 was higher than the average similarity of the group in either year. However, the similarity of individual contextual sequences was less than for the group in either year, 15 percent versus 19 and 24 percent. It seems that individual habit is persistent over time and that individual activity patterns set them apart from the group. On the other hand, context is more variable over time for individuals than it is for larger samples.

\section{Discussion}

\section{Behavioural Patterns}

The activity sequences identified three broad behavioural groups of diaries: employed workers, homemakers, and weekend activity. The activity-setting sequences identified, effectively, the same groups: employed workers, homemakers, and weekend activity, and a residual group with mixed activities. In a sense it may be surprising that more differences were not identified, given 
Evolution of Daily Activity Patterns from 1971 to 1981:

A Study of the Halifax Activity Panel Survey

the additional information provided by the indicators of location and other persons present.

Table 7

Mean Percent Identity Scores for $\mathbf{7 5}$ Halifax Diary Sequences

\begin{tabular}{|c|c|c|c|c|c|}
\hline \multirow[t]{2}{*}{ Comparison } & \multirow{2}{*}{$\mathbf{n}$} & \multicolumn{2}{|c|}{ Activity Sequences } & \multicolumn{2}{|c|}{ Context Sequences } \\
\hline & & Mean & Std Dev & Mean & Std Dev \\
\hline 1971 diaries & 2775 & 39 & 11 & 19 & 12 \\
\hline 1981 diaries & 2775 & 40 & 11 & 24 & 14 \\
\hline Individuals & 75 & 47 & 12 & 15 & 10 \\
\hline
\end{tabular}

A possible reason for the lack of discrimination between the two approaches may be the weighting systems used to define similarity scores. The maximum similarity of 10 was applied to both sets of sequences. The larger number of combinations of matches possible with activity-setting sequences was simply subdivided more finely, depending on the matching patterns at the four positions of each element. There is no theoretical reason to use a constant maximum score, and these results may offer a reason for systematically relating maximum scores to the information content of the sequences.

\section{Behavioural Change 1971-81}

Similarity scores for individual activity sequences for the 1971-81 interval are higher than for the sample averages. It is difficult to say whether or not this might have been expected, ex ante. The people in the panel had lived ten years and had experienced all the growth, decline, joy, sorrow, victory and defeat that time brings. It is clear that key events such as taking or leaving a job occurred and were reflected in the groupings identified by the tree diagrams. Yet the personal behavioural choices made during the period, originating from taste, habit, economic optimization, spirituality, the life cycle or whatever, produced activity patterns more similar than the group average. Individual stability seems to outweigh historical change.

On the other hand, the similarities of individual contextual sequences were less during the period than the group averages in either 1971 or 1981 . We might speculate that context is reflective of the external environment and it changes 
more so than activity. That is to say, individual activities are more under a person's control and subject to choice than the location of activities or services and the presence of other persons when an activity occurs. While the setting is to some extent a matter of personal choice, fundamental changes such as leaving a job, or the death of a spouse will have a permanent influence on the context of a person's activities from their occurrence onward. Such macro-level changes seemed to have affected individual behaviour more strongly than it affects the behaviour of the group.

The increase in the mean similarity for the group from 1971 to 1981 was not expected. Talk of the complexity of modern life, increased personal freedom and so forth, would lead one to expect increasing variability of behavioural patterns. While this particular study is based on a small sample, it points to something to look for in the examination of larger survey samples. Is it possible that activity patterns are becoming less diverse? To the extent that patterns of daily activities represent solutions to the problems of living, is the menu of solutions becoming shorter? If so, does this indicate that undesirable solutions (patterns) are being abandoned or that desirable solutions are no longer achievable? Answers to these questions have applications in a variety of social policy fields including transportation, education, and social services.

\section{References:}

Aas, D. 1978. "Studies of time-Use: Problems and Prospects," Acta Sociologica, 21: 2 .

Allardt, E., 1976. "Dimensions of welfare in a comparative Scandinavian study," Acta Sociologica 19.

Barker, R.G., S. Louise, \& D.D. Ragle. 1967. The Churches of Midwest, Kansas, and Yoredale, Yorkshire: Their Contributions to the Environments of the Towns. In W.J. Gore and L.C. Hodapp (eds.), Change in the Small Community: An Interdisciplinary Survey. New York: Friendship Press.

Chapin, F. S. Jr. 1974. Human Activity Patterns in the City. Toronto: John Wiley \& Sons.

Cullen, I.. and E. Phelps. 1975. Diary Techniques and the Problems of Urban Life. London: Final Report to the Social Science Research Council (Grant No. HR2336).

Durbin, R.. S. Eddy, A. Krough, G. Mitchison. 1998. Biological Sequence Analysis. Cambridge University Press. 
Evolution of Daily Activity Patterns from 1971 to 1981:

A Study of the Halifax Activity Panel Survey

Ellegard, K. 1993. Activities in their everyday context. In Time Use Methodology: Toward Consensus. Rome: Instituto Nazionale d1 Statististics.

Goodhart, D. and A.J. Zautra. 1984. Assessing quality of life in the community. An ecological approach, in W.A. O'Connor and B. Lubin (Eds.). Ecological Models: Applications to Clinical and Community Mental Health. New York: John Wiley \& Sons. Pp.251-290.

Golob, T. F. 1984. Analysing activity pattern data using qualitative multivariate statistical methods, in P. Nijkamp, H. Leitner, and N. Wrigley (eds.) Measuring the Unmeasurable, Proceedings of the NATO Advanced Research Workshop on Analysis of Qualitative Data, Amsterdam, Martinus Nijhoff. Pp.339-356.

Gronmo, S. and E. S. Christensen. 1982. Meaning of time: A field research approach. In Time use studies: Dimensions and applications, Studies no. 128: 89-107.

Hagerstrand, T. 1970. What About People in Regional Science? Papers and Proceedings of the Regional Science Association, XXIV.

Harvey, A. S. 1984. Weekday and Weekend. Chapter 7 in Harvey, A.S., A. Szalai, D. Elliott, P.J. Stone, and S.M. Clark. Time Budget Research, Frankfurt: Campus.

Harvey, A. S. 1982. "Role and Context: Shapers of Behaviour," Studies of Broadcasting, 18: 70-92.

Harvey, A. S. and D. Elliott. 1983. Time and Time Again. Ottawa: Canada Department of Communications Employment and Immigration.

Harvey, A. S. 1997. From Activities to Activity Settings. In Ettema R. and H. Timmermans (eds.) Activity-Based Approaches to travel Analysis. Tarrytown, New York: Pergamon..

Harvey, A. S., D. Elliott, and D. Procos. 1977. Sub-populations relevant to the study of the use of time: A working paper. Regional and Urban Studies Centre, Institute of Public Affairs, Dalhousie University, Halifax, N.S.

Harvey, A. S. and M. Taylor. 2000. "Activity settings and travel behaviour: A social contact perspective," Transportation 27: 53-73

Higgins, D. and P. Sharp. 1988. "CLUSTAL: a package for performing multiple sequence alignment on a microcomputer," Gene 73: 237-244. 
Empirical Research and Applications - Andrew S. Harvey and Clarke Wilson

Jannelle, D.G. and M.F. Goodchild. 1983. "Transportation indicators of spacetime autonomy," Urban Geography 4: 317-337.

John, C. H., T. Arentz and H. Timmermans, 2000. "Activity pattern similarity: a multidimensional alignment on method," Transportation Research B, forthcoming.

Juster, 1985. Conceptual and methodological issues involved in the measurement of time use, in Time, goods and well-being., University of Michigan, Survey Research Center, Institute for Social Research. Pp19-32.

Kalton, G. 1985. Sample design issues in time diary studies, in Time, goods and well-being. University of Michigan, Survey Research Center, Institute for Social Research. Pp 93-112.

Koppelman, F. S. and E.I. Pas. 1984. Travel-activity behaviour in time and space: Methods for representation and analysis, in P. Nijkamp, H. Leitner, and N. Wrigley (eds.) Measuring the Unmeasurable, Proceedings of the NATO Advanced Research Workshop on Analysis of Qualitative Data, Amsterdam, Martinus Nijhoff. Pp. 587-627.

Kutter, E. 1973. "A model for individual travel behaviour," Urban Studies 10(2): 235-258.

Lewin, K. 1951. Field Theory in Social Science. New York: Harper \& Row.

Lynch, K. 1972. What Time is This Place? Cambridge: Mass., MIT Press.

Merz, J. and T. K. Rauberger. 1992. Time Use Questions in a Panel DesignExperiences with the German Socio-Economic Panel. Paper presented at the International Association for Time Use Research, June 15-18, 1992.

Michelson, W. 1972. Environmental choice: A draft report on the social basis of family decisions on housing type and location in Greater Toronto, Discussion paper B.72.9. Ministry of State for Urban Affairs, Ottawa.

Moore, W. E. 1963. Man, Time and Society. New York: Wiley

Needleman, S. and C. Wunsch. 1970. "A general method applicable to the search for similarities in the amino acid sequence of two proteins," Journal of Molecular Biology 48: 443-453. 
Evolution of Daily Activity Patterns from 1971 to 1981:

A Study of the Halifax Activity Panel Survey

Pas, E. 1983. "A flexible and integrated methodology for analytical classification of daily travel-activity behaviour," Transportation Science 17: 405-429.

Pas, E. 1988. "Weekly travel activity behavior," Transportation_15: 571-581.

Recker, W., M. McNally and G. Root. 1985. "Travel/activity analysis: Pattern recognition, classification and interpretation," Transportation Research A, 19: 279-296.

Robinson, J., R. Kitamura, and T. Golob. 1992. Daily travel in the Netherlands and California: A time-diary perspective. Hague Consulting Group.

Sankoff, D. and J. Kruskal (eds.). 1983. Time Warps, String Edits and Macromolecules: The Theory and Practice of Sequence Comparison. Reading MA: Addison-Wesley.

Schneider, A. 1972. Patterns of social interaction. In Szalai A. (ed.) The Use of Time. The Hague: Mouron.

Thompson, J., D. Higgins, and T. Gibson. 1994. "CLUSTALW: improving the sensitivity of progressive sequence alignment," Nucleic Acids Research 22: 4673-4680.

Waterman, M. 1995. Introduction to Computational Biology, Chapman and Hall, London

Wilson, C. 1998a. "Activity pattern analysis by means of sequence alignment methods," Environment and Planning A, 30: 1017-1038.

Wilson, C. 1998b. "Analysis of travel behaviour using sequence alignment methods," Transportation Research Record No. 164.

Wilson, C. 1999. Sequence alignment analysis of daily activities, in J. Merz and M. Ehling (eds.). Time Use - Research, Data and Policy. Nomos, Baden-Baden. (Contributions from the International Conference on Time Use, University of Luneburg, Germany)

Wilson, C. 2001. Activity patterns of Canadian women: an application of ClustalG sequence alignment software. Paper presented at the Transportation Research Board 80th Annual Meeting. Paper 01-2292.

Zuzanek, J. 1979. Evaluation of the instruments used in leisure studies: Canada 1972, 1975, 1976 and 1978. Suggestions for further research. University of Waterloo, Department of Recreation and Department of Sociology. 
Empirical Research and Applications - Andrew S. Harvey and Clarke Wilson

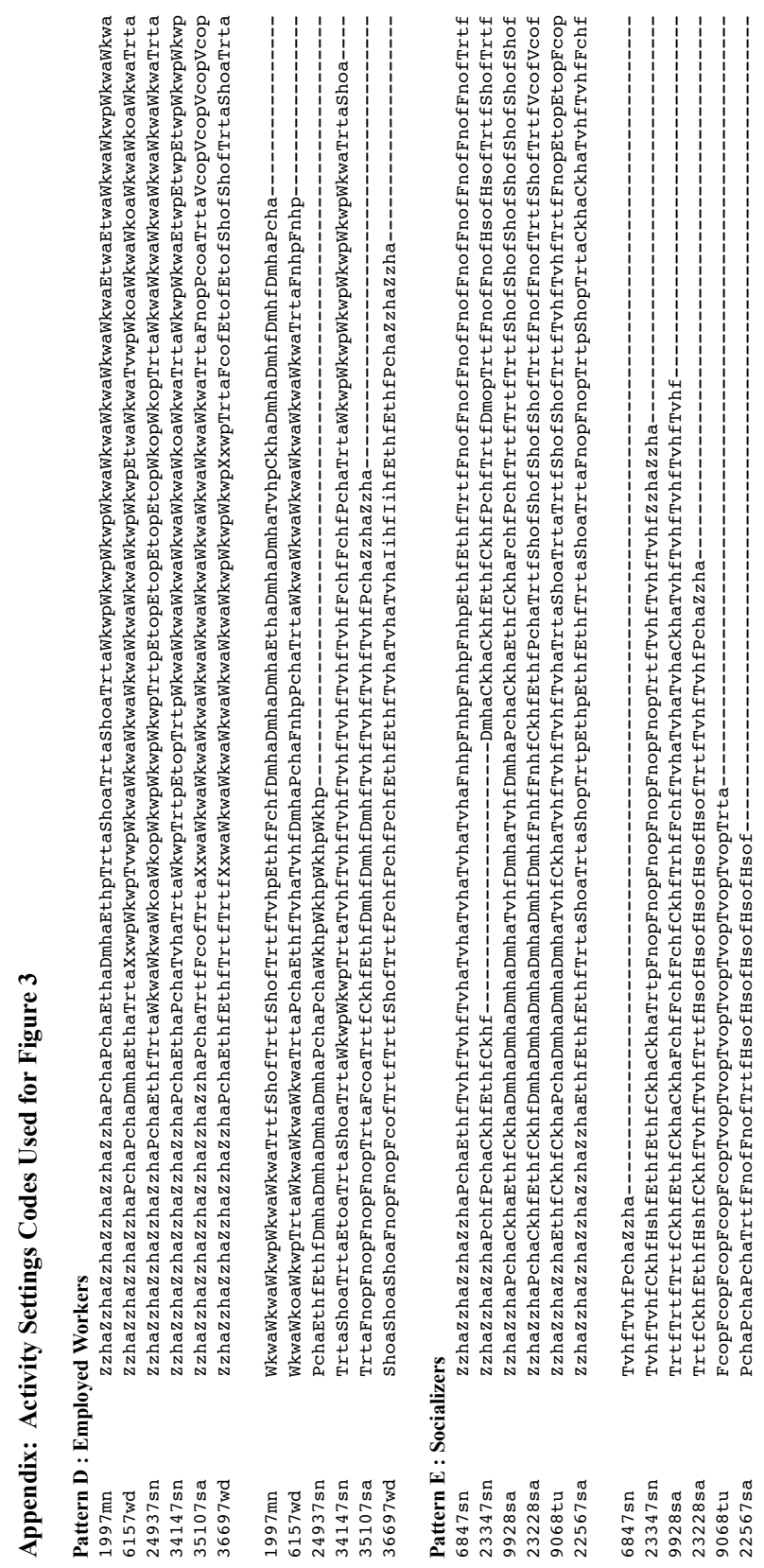


Evolution of Daily Activity Patterns from 1971 to 1981: A Study of the Halifax Activity Panel Survey

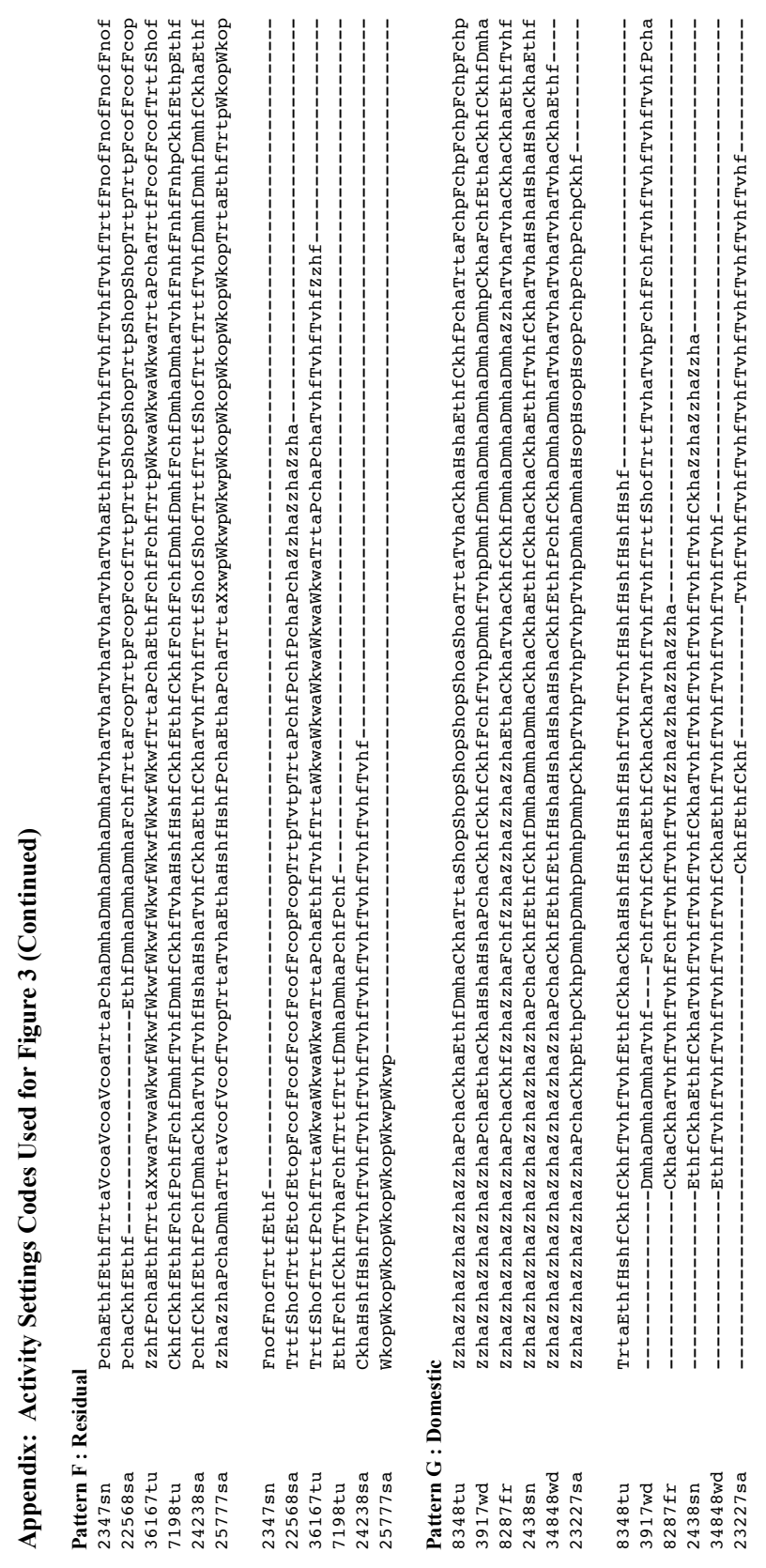

